\title{
Sustentabilidad y economía: la controversia de la valoración ambiental
}

\section{Sustainability and economics: controversy on the environmental valuation}

\author{
Alma Angelina Haro-Martínez \\ Isabel Cristina TADDEI-Bringas*
}

\begin{abstract}
In the topic of sustainable development, the assignment of value to ecosystem services is controversial. The economic science approaches it from two different perspectives: the traditional framework of neoclassical economy (environmental economics), and from an integral viewpoint where different sciences converge: ecological economics. This paper presents a critical review of the conventional economic theory related to sustainable development and highlights the need to assign values to ecosystem services for a comprehensive decision-making process which take into account individual and social preferences, and this way, advance to reach the central goals of sustainable development: economic efficiency, social justice and ecological sustainability.
\end{abstract}

Keywords: sustainable development, ecological economics, environmental economics, valuation.

\section{Resumen}

En el tema del desarrollo sustentable una controversia fundamental es la asignación de valor a bienes y servicios ambientales. La economía lo aborda desde dos perspectivas: el enfoque tradicional neoclásico (economía ambiental) y otra, más integral, donde confluyen distintas ciencias (economía ecológica). Este trabajo ofrece una revisión crítica de la teoría económica convencional asociada al desarrollo sustentable, asimismo, destaca la necesidad de asignar valores a los servicios ambientales para una mejor toma de decisiones, en la que se consideren las preferencias individuales y las sociales, con lo que avanzaría hacia las metas centrales del desarrollo sustentable: eficiencia económica, justicia social y sustentabilidad ecológica.

Palabras clave: desarrollo sustentable, economía ecológica, economía ambiental, valuación, valoración.

*Centro de Investigación en Alimentación y Desarrollo, A.c. Correos-e: haro.alma@gmail.com, ctaddei@ciad.mx 


\section{Introducción}

Uno de los mayores retos que enfrenta actualmente la humanidad es la convivencia armónica con la naturaleza. Es imposible concebir al ser humano independiente de los recursos que el medio ambiente le proporciona; su alimentación, y todos los insumos materiales que sostienen la producción de bienes y la misma vida, está soportada en los ecosistemas de la tierra, de ahí proviene la importancia de conservarlos.

Por otra parte, las demandas de la población van más allá de cubrir sus necesidades básicas, incluyen la mejora en niveles de confort y, en algunos sectores, la acumulación de riqueza. Esto, aunado a la administración inadecuada de los recursos naturales, ha ocasionado la alteración de prácticamente todos los ecosistemas y la consecuente afectación del bienestar del ser humano.

Cualquier intento por explicar este proceso nos remite a la revisión de los orígenes del desarrollo, en cuya evolución han surgido respuestas orientadas a mejorar el bienestar social sin deteriorar los recursos, como las que ofrece el paradigma del desarrollo sustentable.

Alrededor de esta aspiración o principio ético se han construido desarrollos teóricos que retoman algunos postulados básicos de la ciencia económica. La sustentabilidad es abordada, fundamentalmente, a través de dos enfoques: la economía ambiental, basada en el pensamiento económico neoclásico; y la economía ecológica, que constituye una perspectiva ecléctica al retomar premisas de distintas ciencias como la economía, biología, ecología y sociología.

El primer enfoque se distingue por su propuesta basada en la existencia de derechos de propiedad y en la necesidad de asignar precios a todos los bienes y servicios, incluyendo a la naturaleza y los derivados de ésta, en tanto que el enfoque heterodoxo (economía ecológica) cuestiona la conmensurabilidad a ultranza.

El objetivo de este artículo es analizar, a partir de la revisión de los principios del desarrollo sustentable y de las teorías económicas asociadas, la factibilidad de asignar valores a los bienes y servicios de los ecosistemas y con ello aportar elementos para la discusión acerca de la controversia existente entre las diversas disciplinas involucradas en la conmensurabilidad de la naturaleza.

\section{Hacia una definición del desarrollo sustentable}

El modelo de desarrollo que ha prevalecido por más de un siglo ha modificado el carácter de dependencia del ser humano con la naturaleza. Este modelo ha derivado en una fuerte presión sobre los recursos naturales que 
se evidencia en la degradación ambiental, caracterizada por la erosión, desertificación, deforestación, pérdida de biodiversidad y suelos, así como por la contaminación de aguas, aire y suelos, generando diversos problemas sociales como pobreza, desnutrición, desempleo y violencia.

La degradación surge desde que el ser humano empieza a utilizar procesos tecnológicos altamente consumidores de energía en la agricultura, pero se intensifica a partir de la revolución industrial, lo que acarrea una consecuente problemática social (Costanza et al., 2014). En esa época, la abundancia relativa de recursos y el hecho de que la escasez ya no se limitaba tanto a la tierra sino al capital, propiciaba la posibilidad de que la economía hiciera abstracción del mundo físico y lo redujera únicamente a aquellos objetos de valor utilitario para el ser humano.

Tanto los especialistas como la sociedad llegaron a concebir un mundo sin límites físicos, el crecimiento económico se volvió el objetivo primordial y lo indispensable ya no era la satisfacción de las necesidades humanas sino la acumulación de la riqueza, lo que generó una dinámica de apropiación de recursos naturales (Costanza, et al., 2014).

Surgió entonces un modelo de desarrollo económico productivista, cuyos rasgos son aún vigentes, que tiene como premisa fundamental el crecimiento económico. De acuerdo con este paradigma, cualquier problemática puede ser resuelta con el avance tecnológico (Gómez-Baggethun et al., 2010). Las relaciones del hombre con la naturaleza están basadas en una ética antropocéntrica, es decir, el ser humano es el único con derecho a decidir qué tiene valor, el cual se establece en función de la utilidad que proporciona (Azqueta, 2002).

Hacia la década de los sesenta del siglo pasado, el crecimiento económico era el principal objetivo de los países; mayor desarrollo debía implicar mayor bienestar. Sin embargo, aun con el crecimiento de los indicadores económicos, los problemas sociales, al igual que los relativos a la degradación de los recursos naturales, iban en aumento. Se evidenciaba que el sistema existente promovía la desigualdad económica y social, así como dańos a los recursos de la biosfera poniendo en peligro su permanencia. En los setenta, diversos analistas empezaron a insistir en la necesidad de plantear un nuevo modelo de desarrollo.

En 1987, la Comisión Mundial del Medio Ambiente y Desarrollo (World Commission on Environment and Development) propuso la integración de la conservación del medio ambiente a los objetivos del desarrollo social y económico bajo la etiqueta de desarrollo sustentable a través del informe Brundtland Nuestro futuro común, que lo definió como "aquel que satisfaga las necesidades del presente sin comprometer la capacidad de las futuras generaciones para satisfacer las propias" (Brundtland, 1987). 
La necesidad de integrar en el análisis los procesos económicos, ecológicos y sociales ha exigido la atención de diferentes disciplinas, lo que ha derivado en diversas definiciones $y$, aunque persisten divergencias en cuanto a los contenidos, muchas de ellas se enfocan en el sentido ético y moral del desarrollo. Algunas tienen como prioridad incidir sobre el bienestar, otras propugnan más por la defensa de la sustentabilidad ecológica.

Sin embargo, y de acuerdo con los fundamentos de este enfoque, la mayoría asume que el desarrollo sustentable debe guardar equilibrio en tres dimensiones básicas: la social, la económica y la ecológica o ambiental, lo que permitiría cumplir con la finalidad ética y social del desarrollo, y con una disposición y gestión más eficiente de los recursos, donde se aplicaría la la planificación social y el uso adecuado de los recursos ambientales, además, se mantendrían los sistemas de soporte a la vida.

Los principios del desarrollo sustentable son enfoque integrado o con visión interdisciplinaria y solidaridad con las generaciones futuras, debe ser equitativo y justo; se requiere de una participación conjunta de la población que permita incidir en la toma de decisiones; también debe considerarse la capacidad de regeneración y asimilación del planeta, de ahí la necesidad de la conservación de los ecosistemas.

Los límites del desarrollo actual no se encuentran en la tecnología y la cantidad de recursos existentes, tienen que ver con la desigualdad social, la intensidad en el uso de los recursos naturales y la capacidad del planeta para absorber los residuos resultantes de la producción y consumo. El proceso de desarrollo sustentable como tal se sustenta en una economía saludable con equidad social y calidad ambiental; sus objetivos han de enfocarse en eliminar la pobreza y mantener la economía en su tamaño ecológico óptimo, es decir, regular la tasa de uso de los recursos para que ésta no rebase su índice de crecimiento o reposición.

El desarrollo sustentable, en tal sentido, va más allá de ser un principio de carácter científico-técnico, es un principio normativo que pretende que la vida humana pueda perpetuarse. De acuerdo con Costanza et al. (2014), es un concepto ético filosófico que se apoya en distintas ciencias para explicar y lograr sus fines; sus planteamientos demandan el manejo de sistemas complejos, ya que se requieren soluciones a problemas globales de largo plazo que involucran a muchas disciplinas y conexiones entre éstas.

\section{Economía ambiental y economía ecológica}

Las distintas teorías del desarrollo han tenido como base las doctrinas económicas, de ahí que sea imposible separarlas de la dimensión económica (Jiménez-Herrero, 2003). El desarrollo sustentable utiliza las herra- 
mientas de la economía para operativizarse, es decir, para poner en práctica los elementos que permitan alcanzar esta aspiración o principio ético, lo que hace a través de dos aproximaciones o enfoques: la economía ambiental y la economía ecológica.

De acuerdo con algunos autores, como Jiménez-Herrero (2003), este tipo de desarrollo va más allá de los parámetros de la economía convencional y se adapta mejor al enfoque de la economía ecológica. A continuación se describen ambos enfoques, los cuales tienen como fin la integración de la naturaleza a la economía, aunque difieren en la forma de hacerlo.

\subsection{Economía ambiental}

Hacia finales de la década de los cincuenta y principios de los sesenta del siglo pasado, ante el deterioro de la naturaleza atribuido a la evolución de la economía, la ciencia económica reconoció, en el debate sobre el desarrollo, que los recursos naturales podían constituir una restricción para el crecimiento, por lo que era necesaria una nueva relación entre los recursos físicos y la economía (Naredo, 2001); es así como surge la economía ambiental, con este nuevo enfoque teórico se incorporaba la variable naturaleza al mercado sin renunciar por ello al objetivo de crecimiento económico.

De acuerdo con Riechmann (1995), la biosfera desempeña tres funciones indispensables e insustituibles: fuente esencial de la vida y hábitat para las especies vivas, almacén de energía y materias primas y vertedero de desperdicios. Sin embargo, bajo el paradigma económico predominante se asume que las materias primas provenientes del ambiente son inagotables y los productos de desecho irrelevantes. Esa idea propicia que los servicios proporcionados por el medio ambiente no cuenten con un precio, debido a que no existen mercados específicos para su intercambio (Van-Dyke, 2008).

Según este enfoque, la inexistencia de mercados en este tipo de bienes y servicios se explica por la ausencia de derechos de propiedad bien definidos como sucede en el caso de los bienes públicos (el aire que respiramos o la capa de ozono) y los recursos comunes (los bosques abiertos o acuíferos no regulados), ambos se caracterizan por la no exclusión, es decir, no puede excluirse a nadie de su disfrute, sin embargo, se diferencian porque en el primero no existe rivalidad en el consumo, pues su uso no reduce su disponibilidad, mientras que en el segundo sí (Carciofi y Azqueta, 2012).

El problema con los recursos comunes se presenta cuando son de libre acceso, lo que implica que su uso no tiene ningún costo, pero a diferencia 
de los bienes públicos, su agotamiento o degradación es posible debido a la rivalidad en el consumo y a la falta de regulación. El mercado no proporciona ningún indicador del valor de los recursos ambientales, de ahí que en muchos casos éstos llegan a considerarse como gratuitos en virtud de que su uso y disfrute carecen de algún costo monetario. Cuando esto sucede, el costo de apropiación de un bien o degradación de un servicio es pagado por la sociedad; esto es, se convierten en subsidios ocultos que impiden percibir la importancia de la conservación de los recursos.

El sistema económico considera esta situación como una externalidad porque se da cuando la actividad de una persona repercute sobre el bienestar de otra sin que ésta pueda cobrar un precio por ello en caso de ser negativa, o pagarlo, en caso de ser positiva (Azqueta, 2002). La economía ambiental propone convertir esas externalidades negativas en una escala que sea comparable con los elementos del sistema económico; para ello, plantea el uso de algunas técnicas que permitan asignar un precio a los bienes y servicios ambientales lo cual resultará útil al momento de diseñar instrumentos específicos como impuestos, subsidios; o bien, para su integración directa al precio. De esta manera, se internalizarían esas externalidades negativas y se desincentivarían las actividades o acciones degradatorias al ambiente. La valoración ambiental es la herramienta que permite dicha asignación.

A la economía ambiental se le ha conocido como la economía neoclásica de los recursos naturales y de la contaminación ambiental, se considera una extensión de la economía convencional (Martínez-Alier et al., 1998). De acuerdo con algunos autores, como Aguilera-Klink y Alcántara (1994), este enfoque no constituye una crítica al sistema económico actual sino que representa una especialización dentro de la economía neoclásica. De acuerdo con Azqueta (2002), no se trata de que la economía ambiental intente que el mercado defina la calidad del ambiente con que la sociedad desea convivir, sino de utilizar la lógica del mercado para descubrir el valor del mismo y hacia dónde desearía encaminarse la sociedad en términos de la calidad ambiental.

\subsection{Economía ecológica}

La economía ecológica surge como una crítica a los planteamientos de la economía ambiental, pone en duda parte del instrumental de la economía convencional en que se basa, ante las evidencias de su fracaso. Pretende profundizar en aquellos aspectos sociales y ecológicos que la economía convencional no ha considerado, al integrar los procesos económicos y ecológicos con base en criterios de eficiencia y equidad (Jiménez-Herrero, 2003). 
La economía ecológica nace propiamente como rama de la economía en la década de los ochenta del siglo pasado, al suscitarse divergencias teóricas en la economía ambiental relativas al papel de la sustituibilidad y monetarización de los recursos naturales (Costanza et al., 2014; GómezBaggethun, et al., 2010).

Las primeras contribuciones parten de la crítica a la teoría neoclásica realizada hace más de cien años por Sergei Podolinksy, Patrick Geddes y Fredrick Soddy, quienes intentaron incorporar análisis de flujos de energía en los estudios económicos (Tetreault, 2008); posteriormente, Nicolas Georgescu-Roegen (1971) propone su obra The entropy law and the economic process la cual, a pesar de las críticas, constituyó la base del cambio de paradigma. En esta obra se señala que la degradación de la energía, a través del fenómeno de la entropía, es irreversible, postulado que se convierte en el fundamento teórico principal de este enfoque (Herrmann-Pillath, 2011).

Desde esa perspectiva, la economía depende de la presencia de los ecosistemas para su existencia, de ahí que la sobrevivencia de los procesos físicos y biológicos tiene prioridad sobre la rentabilidad, independientemente de la percepción de la gente; tiene como propósito el estudio de todos los objetos que componen la biosfera y los recursos naturales, que no necesariamente proporcionan alguna utilidad a las sociedades humanas: el valor de los recursos ambientales es intrínseco a ellos.

La economía ecológica se centra en la naturaleza física de los recursos y su vínculo con los sistemas que se interrelacionan; toma en cuenta desde la escasez y la renovabilidad de los mismos hasta la nocividad y el posible reciclaje de los residuos generados; tiene el propósito de orientar el marco institucional y generar propuestas de solución (Naredo, 1994).

Dada la complejidad de las interrelaciones dinámicas entre el sistema económico y los sistemas físico y social, este enfoque busca estudiar los problemas de manera transdisciplinaria, para construir respuestas a la problemática ambiental y social.

A partir de la convergencia de distintas disciplinas, se han desarrollado diversas aportaciones a la teoría económica que llevan a sostener que la economía ecológica propone, más que modificaciones al sistema económico predominante, una reconstrucción conceptual de la economía (Kapp, 1976). ${ }^{1}$

La economía ecológica es considerada como el paradigma actual y como la ciencia de la gestión de la sostenibilidad (Costanza, 1991); esta parte de la premisa de que el ecosistema global (la tierra) es finito y tiene

\footnotetext{
${ }^{1}$ Las aportaciones de Kapp son anteriores a lo que hoy se conoce como economía ecológica, sin embargo, se considera que éstas constituyen la base de dicho enfoque.
} 
capacidades limitadas de asimilación y regeneración se centra en la interacción de los sistemas económico, social y ambiental en el marco de la equidad, distribución, ética y cultura.

Si se considera que la economía es un subsistema dentro del ecosistema global, abierto al intercambio de energía, materia e información, se asume entonces que está sujeta a las leyes de la naturaleza. Así, la economía ecológica se fundamenta en tres principios biofísicos básicos:

La primera ley de la termodinámica, que afirma que la materia y la energía no se crean ni se destruyen, sólo se transforman; es decir, permanecen constantes en un sistema cerrado (como la tierra). Esto implica que al extraer recursos o utilizar energía éstos deberán regresar de alguna manera, en algún momento y en la misma proporción; por lo tanto, los procesos de producción y consumo siempre irán acompañados de la generación de residuos (Aguilera-Klink y Alcántara, 1994).

La segunda ley de la termodinámica, o ley de la entropía, afirma que materia y energía se degradan continuamente en un sentido, de utilizable a inutilizable, o de disponible a no disponible. Por lo tanto, un aumento en la entropía equivale a una disminución de la energía disponible. Cada vez que se lleva a cabo un proceso es disipada cierta cantidad de energía, la cual es irrecuperable y por lo tanto no estará disponible para realizar algún trabajo futuro. Así, lo que confiere valor a la materia o energía es su disponibilidad para ser utilizada. Las principales fuentes de energía en nuestro planeta son hidrocarburos fósiles que al utilizarse pierden la posibilidad de ser reutilizados, lo que implica su agotamiento inminente (Aguilera-Klink y Alcántara, 1994).

El tercer principio se refiere a la imposibilidad de extraer más recursos de los que la naturaleza puede regenerar y de generar más residuos de los que la naturaleza puede asimilar.

De esta manera, el sistema global está limitado por las leyes de la termodinámica; el mundo es finito, lo que implica el agotamiento irreversible de los recursos y de los servicios que éstos prestan. Es por ello que el grado hasta el cual debe operar la actividad económica estará definido por la escala, es decir, por la capacidad de los ecosistemas para soportarla. Uno de los elementos que diferencia a la economía ambiental de la ecológica es, precisamente la escala.

La economía ecológica pone énfasis en los tiempos de producción de la naturaleza, en contraste con los tiempos de producción del sistema económico. Esto es, la destrucción de la naturaleza se expresa en la diferencia entre el tiempo económico y el tiempo biológico, controlado este último por el ritmo de la naturaleza (regeneración de recursos y absorción de residuos). Si se introducen al mercado los bienes y servicios de la naturaleza se alterará esta diferencia, lo cual redundará en una 
elevada tasa de deterioro, donde aparentemente el tiempo económico triunfa sobre el tiempo ecológico (Martínez-Alier, 1996); por ello algunos autores, dentro de este mismo enfoque, desaprueban la utilización de la valoración monetaria.

Autores como Ehrlich y Ehrlich (1993) han considerado que el principal problema ecológico es la sobrepoblación, postura que ha sido muy criticada por quienes plantean que asumirlo así indicaría que los recursos naturales son insuficientes para satisfacer a la población; esta crítica carecería de sentido ante el hecho real de que, aun cuando se trate de recursos abundantes, su uso indiscriminado terminaría por acabarlos.

El verdadero problema entonces se halla en el consumo, pues aunque las necesidades humanas son finitas y deberían ser similares para cualquier ser humano, las sociedades las han modificado en función de su condición histórico-cultural. Sin embargo, para algunos autores, la disminución del consumo implicaría el decrecimiento de la economía y por ende el colapso del sistema (Kallis et al., 2012). La propuesta de Costanza et al. (2014) plantea explorar modelos donde la planeación económica, ecológica, social y política sea central y el problema de los bienes sustitutos no se resuelva con encontrar nuevos estilos de vida, sino a través de la reconfiguración de éstos, particularmente de quienes obtienen mayores ingresos; asimismo, sería necesario, además, considerar a la sustentabilidad como indispensable para la subsistencia.

De esta manera, la economía ecológica se puede considerar una crítica a la economía convencional, ya que incorpora una nueva forma de visualizar y asumir las relaciones entre los sistemas físico y social; además, su carácter transdisciplinario permite entender las interrelaciones de sistemas complejos y visualiza a la naturaleza como conjunto ordenado de ecosistemas que requieren conocerse ampliamente para poder orientar la gestión. Se basa en el conocimiento físico de la biosfera para sustentar la valoración monetaria y la toma de decisiones de los agentes económicos.

Sus fundamentos son la equidad, la ética y la justicia, valores que retoma para comprender la sustentabilidad y propone medirla a través de indicadores biofísicos como complemento a los monetarios. Incorpora en el análisis aquellos aspectos que pueden quedar ocultos por el sistema de precios, lo que implicaría subvaluar los recursos y procesos ambientales así como sus repercusiones.

\subsection{Contrastes y concordancias entre ambos enfoques}

Mientras que la economía ambiental construye la valoración únicamente sobre los objetos que proporcionan bienestar a la sociedad o le son útiles, la economía ecológica extiende el análisis al resto de objetos económicos 
que no pueden traducirse a unidades monetarias y complementa la valoración con la medición física de los recursos en los casos donde los valores monetarios no resultan válidos.

Según autores que se adscriben al paradigma de la economía ecológica, como Naredo (2001) y Martínez-Alier y Roca-Jusmet (2001), la economía ambiental incorpora la transformación del entorno natural como un problema de asignación de recursos escasos y fomenta su monetarización. Esto contrasta con el postulado de la segunda ley de la termodinámica que plantea que toda transformación material es irreversible y da lugar a la generación de desechos físicos y energía disipada.

Además, consideran que la valoración monetaria de las externalidades es aplicable solamente en el caso de fenómenos reversibles (como la contaminación del agua, que se puede limpiar), pero en el caso de los irreversibles (como la extracción y agotamiento de un recurso) o donde se involucró un procesamiento o disipación de energía, no procede la asignación de valor.

Otras críticas desde la economía ecológica señalan que el enfoque de la economía ambiental asume que la naturaleza está constituida únicamente por el capital natural, con lo que deja de lado las funciones complejas surgidas de la interrelación de ese capital con flujos de energía a los cuales no puede asignárseles valor. Una crítica central es que, aun cuando sean muy altos los valores monetarios asignados, ello no evita el deterioro.

Por otro lado, en lo que tal vez constituye la mayor diferencia entre ambos enfoques, está la medición de la escala a la hora de asignar valores, ya que mediante ésta se concede importancia a la capacidad de asimilación y regeneración del sistema evaluado. La propuesta de la economía ecológica es tomar como referencia una escala sustentable, es decir, que no erosiona la capacidad de carga ambiental con el paso del tiempo.

La economía ambiental ha considerado la incorporación del entorno natural como un problema de asignación de recursos, sin embargo no contempla las leyes físico-químicas de la naturaleza. Como se ha señalado, la segunda ley de la termodinámica establece la degradación irreversible de la materia y energía que da lugar a desechos físicos y energía disipada, esto implica que en definitiva no se trata sólo de un problema de equidad, sino de degradación y pérdida de recursos energéticos. En este marco, la generación de residuos deberá ser menor a la capacidad de asimilación de los ecosistemas y la extracción menor a su rendimiento sostenible o renovable, lo cual difícilmente ocurre en la actualidad.

Por otra parte, la economía ambiental implica cambios inducidos al intentar incorporar los aspectos ambientales en la toma de decisiones, lo que significa una estrategia de arriba hacia abajo, mientras que la econo- 
mía ecológica conlleva modificaciones que vienen desde la sociedad, lo que supone una estrategia de abajo hacia arriba (Tetreault, 2008).

De acuerdo con el paradigma de la economía ecológica, la tecnología debe armonizarse con el desarrollo sostenible y, de hecho, los avances en ese campo son necesarios para la generación de tecnologías limpias orientadas a aumentar la productividad de los recursos; es decir, generar mayor volumen por unidad de recurso, así como incursionar en el diseño de productos y de procesos que faciliten el reciclaje (Daly, 1996).

En contraste, la economía ambiental considera que el uso de recursos no tiene que ser limitado, ya que una vez que éstos se vuelvan escasos se dará lugar a innovaciones tecnológicas orientadas a satisfacer las necesidades humanas (Illge y Schwarze, 2009), Es así como este enfoque asume una perfecta sustituibilidad entre el capital natural y el producido, y precisa de un crecimiento sustentable que considera la no disminución del consumo per cápita a través del uso óptimo de los recursos y la tecnología (Singh et al., 2009; Gómez-Baggethun et al., 2010).

Una de las diferencias más marcadas entre los dos enfoques tiene que ver con la territorialidad. En el caso de la economía ecológica los datos que se obtengan para la asignación de valores deben estar vinculados con un territorio de referencia, de ahí que sea indispensable el uso de herramientas cartográficas tales como los sistemas de información geográfica, mientras que en el enfoque convencional, centrado en la racionalidad, se concibe un mundo sin dimensiones que evoluciona al margen de las referencias territoriales concretas y considera las cifras como los instrumentos de trabajo más adecuados (Naredo, 1996). En el cuadro 1 se sintetizan algunas de estas diferencias.

\section{Cuadro 1}

Diferencias significativas entre la economía ambiental y la economía ecológica

\begin{tabular}{|c|c|}
\hline Economía ambiental & Economía ecológica \\
\hline Ética antropocéntrica. & Ética de la tierra o biocéntrica. \\
\hline Objetos útiles. & $\begin{array}{l}\text { Objetos que componen la biosfera y los } \\
\text { recursos naturales. }\end{array}$ \\
\hline Sistema cerrado en equilibrio. & Sistema abierto en desequilibrio. \\
\hline Enfoque reduccionista. & Enfoque de sistemas. \\
\hline Crecimiento ilimitado. & $\begin{array}{l}\text { Crecimiento demarcado por los recursos } \\
\text { naturales y la tecnología. }\end{array}$ \\
\hline $\begin{array}{l}\text { Sustituibilidad entre capital natural } \\
\text { y manufacturado. }\end{array}$ & $\begin{array}{l}\text { Complementariedad entre ambos tipos de } \\
\text { capital. }\end{array}$ \\
\hline
\end{tabular}


continúa...

\begin{tabular}{|c|c|}
\hline Economia ambiental & Economía ecológica \\
\hline $\begin{array}{l}\text { Valoración monetaria sólo a objetos útiles } \\
\text { (valor instrumental). }\end{array}$ & $\begin{array}{l}\text { Valoración monetaria basada en valores } \\
\text { intrínsecos y complementada con valora- } \\
\text { ción a partir de indicadores biofísicos. }\end{array}$ \\
\hline Corto plazo. & Largo plazo. \\
\hline Estrategia de arriba hacia abajo. & Estrategia de abajo hacia arriba. \\
\hline $\begin{array}{l}\text { Tecnologías que incrementan la cantidad } \\
\text { de recursos. }\end{array}$ & $\begin{array}{l}\text { Tecnologías que incrementan el volumen } \\
\text { por unidad de recurso. }\end{array}$ \\
\hline Asignación y distribución. & $\begin{array}{l}\text { Asignación eficiente, distribución justa y } \\
\text { escala sustentable. }\end{array}$ \\
\hline Cifras sin asignación territorial. & Territorialidad. \\
\hline
\end{tabular}

Fuente: elaboración propia.

A pesar de las diferencias entre ambos enfoques es importante admitir que tienen como objetivo común el conocer y entender la interacción ser humano-economía-medio ambiente, que conduzca a la economía hacia la sustentabilidad (Venkatachalam, 2007).

\section{Del desarrollo sustentable conceptual al operativo}

\subsection{La valoración}

El valor se define, en general, como el grado de utilidad o aptitud de las cosas para satisfacer las necesidades o proporcionar bienestar o deleite. Los seres humanos le conferimos valor a distintos elementos en función de nuestra concepción de las necesidades que satisface el objeto valuado. $\mathrm{Si}$ consideramos que el medio ambiente proporciona una amplia gama de valores que afectan de forma positiva el bienestar, entonces podemos decir que adquiere un valor para la sociedad (Azqueta, 2002). Según la economía ambiental son estos valores, dado su carácter utilitario, los que pueden ser integrados al sistema económico.

Sin embargo, más allá de su aprovechamiento, el verdadero valor de los ecosistemas está en su capacidad para cumplir importantes funciones ecológicas y ambientales que, aun cuando no tengan un valor utilitario, son fundamentales para la vida y proporcionan bienes y servicios indispensables para satisfacer las necesidades del ser humano y su desarrollo; esta capacidad se ha visto mermada por la intervención del mismo ser humano. Si bien el carácter renovable permite a los ecosistemas restablecerse, las necesidades de la población han superado el tiempo requerido para hacerlo posible, por lo que la calidad y cantidad de los servicios que proporcionan han disminuido a tal grado que en algunos casos su alteración es irreversible. 
La valoración ambiental surge como una herramienta de la economía ambiental que considera útil valorar en términos monetarios los servicios que proporcionan los ecosistemas, así como el impacto sobre ellos. Es decir, se intenta asignar valores cuantitativos a los bienes y servicios proporcionados por los recursos ambientales, independientemente de si existen o no precios de mercado que permitan hacerlo; ello, con el fin de generar indicadores que den cuenta de la importancia de dichos recursos para la sociedad (Willis et al., 1999). De acuerdo con los autores principales de este paradigma, el hecho de que los servicios sean intangibles y existan problemas para evaluarlos en términos físicos no significa que sea imposible hacerlo en términos monetarios.

Quienes sostienen la conveniencia del uso de esta herramienta, si bien consideran que algunos elementos del medio ambiente son invaluables, afirman que al no valuarlos se caería en el error de asumir que su valor es cero y así se excluirían de la toma de decisiones, de ahí la necesidad de estimar los valores para tener elementos que permitan comparar los factores ambientales con los sociales y económicos, aun cuando se corra el riesgo de asignar valores menores a los reales.

\subsection{Valoración monetaria y conmensurabilidad}

La valoración es el proceso de estimar y expresar un valor para una acción u objeto en particular; en el caso del medio ambiente, este proceso se enfoca a la asignación de valor monetario a bienes y servicios de un ecosistema.

Se trata de una herramienta controversial, ya que existen diversos cuestionamientos a la asignación de un precio a la naturaleza; se argumenta la dificultad o imposibilidad de medir su valor intrínseco además de la incapacidad de asignar valores a costos sociales futuros, debido a la incertidumbre de los procesos. Sin embargo, de acuerdo con el enfoque de la economía ambiental la valoración monetaria constituye un instrumento indispensable para avanzar hacia el equilibrio entre economía y medio ambiente.

Para la economía ecológica, en cambio, es necesario pasar de los valores de mercado a un tipo de éstos que reflejen el valor intrínseco de la naturaleza, con base en su existencia misma y en los servicios ambientales que proporciona; de esta manera, se superaría la visión de corto plazo para trascender a las generaciones futuras.

A pesar de las diferencias en las apreciaciones de estos enfoques respecto al valor, es evidente que en ambos se utilizan, aunque con una visión distinta (Spash, 2013). Autores como Castro e Silva y Teixeira (2011) 
sugieren que el cambio social en cuanto a la relación con el medio ambiente estará fuertemente vinculado a los valores.

Es posible encontrar una gran cantidad de índices para medir diversos aspectos de la sustentabilidad (Ness et al., 2007; Singh et al., 2009), sin embargo, de acuerdo con Gasparatos y Scolobig (2012), al utilizar indicadores compuestos no es posible hacer una valoración específica sobre el rol de los seres humanos, al igual que sucede con los indicadores biofísicos o económicos, pues las nociones de valor se pierden durante la normalización y agregación de los elementos que los integran.

En años recientes se han adoptado a escala regional instrumentos basados en el mercado para evaluar la condición de las regiones. Empero, la valoración sigue siendo un gran reto; persiste una brecha amplia de conocimiento acerca de las relaciones biofísicas y eco-hidrológicas que deben servir de base para un modelado confiable.

Identificar los servicios de los ecosistemas proporcionados por una región y evaluar su contribución al bienestar humano es una tarea de enormes proporciones (Maynard et al., 2014). Sin embargo, se avanza en el diseño de instrumentos que consideran la espacialidad e integran la información económica y biofísica disponible y con ello permiten generar valores confiables para estimar el valor integral de territorios o regiones.

Esta valoración no sólo posibilita incorporar tales valores al sistema económico, sino que proporciona información adicional para evaluar cómo éstos varían a través del espacio y, cómo, a través de ellos, es posible incidir en el diseño de políticas que propicien la recuperación e incremento de las capacidades de los servicios ambientales y, consecuentemente, un impacto favorable en el bienestar social (Egoh et al., 2009; Schagner et al., 2013).

De acuerdo con Haro-Martínez y Taddei-Bringas (2010) el uso de Sistemas de Información Geográfica (SIG) para identificar y cuantificar servicios ambientales adquiere cada vez mayor reconocimiento por sus alcances en la identificación espacial o territorial para la estimación de valores confiables y el uso eficiente de recursos, de ahí que se ha convertido en una base de referencia para la adopción de esquemas de Pago de Servicios Ambientales (PSA).

Los esquemas de PSA son la transacción voluntaria de servicios ambientales entre un proveedor y el usuario de los mismos, que permite compensar al primero por la garantía del servicio a cambio de una retribución generalmente económica (Derissen y Latacz-Lohmann, 2013); constituyen la primera aproximación práctica a la integración del sistema económico con el social y el ambiental.

Estos esquemas han cobrado vigencia en los últimos años debido a su potencial para cambiar el comportamiento dañino para el ambiente y por 
los beneficios que puede aportar a los medios de vida rurales (Tacconi, 2012). En la actualidad, la valoración de servicios ambientales ocupa un lugar central en las agendas políticas y académicas de la conservación de la biodiversidad por su utilidad en la toma de decisiones (Laurans y Mermet, 2014).

Aun cuando los enfoques de la economía ambiental y la economía ecológica coinciden en la necesidad de utilizar este instrumento, existen discrepancias respecto a su diseño y aplicación; para la primera representa un esquema para mejorar la eficiencia de la gestión de los recursos naturales, mientras que para la segunda constituye la oportunidad de compatibilizar las decisiones sobre el manejo de recursos naturales con los intereses sociales.

Por las similitudes entre ambas perspectivas, se advierte que es posible una implementación adecuada del esquema de PSA donde, vinculada a la rentabilidad, se considere la equidad inter e intrageneracional (Tacconi, 2012). Ello llevaría a cumplir con las expectativas de ambos enfoques en cuanto a mejorar el manejo de los recursos para asegurar la provisión de los servicios ambientales y de esta manera incidir en el incremento de la calidad de vida y en la reducción de la pobreza, sobre todo en el medio rural.

De acuerdo con Naredo (1994), para la gestión ambiental puede procederse de dos formas: una, desde la valoración monetaria, al aplicar el enfoque analítico de la economía convencional basado en precios, costos y beneficios monetarios; y la otra, adoptando a la gestión los enfoques analíticos de las distintas disciplinas involucradas en la problemática ambiental.

El autor sostiene que ambas perspectivas deben complementarse de tal forma que la utilidad y el bienestar del sistema económico se adapte a las exigencias ecológicas e incorpore las dificultades que representa la interacción con el resto de los sistemas con que se relaciona. Esto implica que el sistema económico se apoye en otros que le proporcionen información adecuada y permitan dimensionar la problemática para incidir en el universo físico en que está inmerso.

De esta forma, se ampliaría el objeto de estudio a otros sistemas y se utilizarían los sistemas que resulten más adecuados al contexto, pasando así del universo del valor monetario a uno donde esté incluida la realidad física, con nuevos modelos predictivos y opciones tecnológicas, y con nuevos procesos de negociación social, donde la discusión se centre no sólo en el mercado, sino en las instituciones y la información.

Como puede advertirse, lo anterior no implica una renuncia a la valoración monetaria, más bien alude a la integración del sistema económico a su entorno biofísico y territorial, dando lugar a una nueva 
estructura de análisis. Este nuevo enfoque llamado ecointegrador lleva a una reestructuración conceptual de la economía al trastocar tanto su instrumental y métodos como sus propios principios (Naredo, 1994).

Por otro lado, desde la economía ecológica no se cuenta con un método de medición estándar; se considera que la evaluación de las externalidades es arbitraria, de ahí que no puede servir como base para el diseño de políticas ambientales. Sin embargo, tampoco es posible que tales políticas se basen solamente en la racionalidad ecológica, ya que para decidir es necesario comparar costos y beneficios, lo que hace indispensable la asignación de valores.

La complejidad que envuelve las interrelaciones de los diversos sistemas involucrados ha llevado a considerar y utilizar la teoría de la decisión multicriterio (Munda, 1995), que propone evaluar todos aquellos criterios necesarios para la toma de decisiones, éstos pueden ser de diversa índole: valoración monetaria, ponderaciones de los distintos factores involucrados, consensos. Para tomar la decisión final se organizan las posibles soluciones en una matriz y se recurre a un juicio práctico basado en la capacidad de percepción y del conocimiento.

Como se advierte, bajo esta lógica no existe una solución única al problema, sino que ésta depende del peso relativo o importancia que se da a cada criterio (Martínez-Alier et al., 1998); lo anterior permite sostener que para una toma de decisiones informada y adecuada dirigida a la solución de problemas complejos se requiere desarrollar instrumentos sólidos, útiles para la evaluación de los distintos elementos involucrados y sus interrelaciones. El método más aceptado y utilizado es el enfoque multicriterio, el cual implica la evaluación individual de cada uno de los elementos, mismos que para ser válidos deben basarse en información confiable; una de las herramientas que pueden proporcionar esta información es la valoración monetaria.

En síntesis, hacer operativo el desarrollo sustentable requiere de la convergencia de las propuestas de ambos enfoques. Tanto las herramientas planteadas por la economía ecológica como las de la economía ambiental deberían complementarse para obtener mejor y mayor información respecto a una toma de decisiones consistente con los principios del desarrollo sustentable: equidad, justicia, eficiencia en el uso de recursos, y que corresponda con la capacidad natural de carga del sistema que se evalúe.

\subsection{Sustentabilidad en la asignación de valores monetarios}

De acuerdo con Naredo (2001), el objeto de estudio de la economía ecológica es la sustentabilidad sin que para ello necesariamente se tenga que recurrir a un solo tipo de valor expresado en números. El otorgar 
valores al ambiente es útil, aunque no necesario; este enfoque no renuncia a la valoración de algunos de los objetos económicos, sino que la limita. Así, algunos de estos objetos tendrán valores monetarios y otros se podrán expresar en unidades físicas.

Es necesario tener en cuenta algunos problemas básicos que aborda la economía ecológica, a partir de los cuales debe realizarse la valoración. Según este enfoque, los retos elementales a superar son escala sustentable, distribución justa y asignación eficiente. La primera se refiere a que la escala o magnitud de las actividades humanas debe ser ecológicamente sustentable; alude a las capacidades naturales del ecosistema para regenerar los insumos y absorber los desperdicios sobre una base sustentable.

La segunda se relaciona con la distribución de los recursos y derechos de propiedad justos, tanto de la presente generación como entre ésta y las futuras generaciones, así como entre humanos y otras especies; aquí, los mecanismos pueden ser los impuestos, los pagos de seguridad social o esquemas de pago por servicios ambientales, entre otros.

La tercera y última sugiere la asignación de recursos entre usos finales del producto (incluye recursos de mercado como los bienes adquiridos y de no mercado como los servicios ambientales) de acuerdo con las preferencias individuales determinadas por la capacidad de pago del individuo. Aun cuando la asignación y distribución son tratadas por la economía convencional, la escala ha sido prácticamente excluida, por lo que es necesario considerar tanto la asignación como la distribución en torno a la sustentabilidad ecológica (Costanza et al., 2014; Costanza y Folke, 1997).

Lo anterior tiene relevancia en el marco de la valoración, desde la economía ecológica, dado que este enfoque se opone a la internalización de los costos externos a través de los precios de mercado, porque ello implica efectos indeseados en la distribución del ingreso. Trasladar a la sociedad las externalidades por medio del mercado conlleva injusticia social, puesto que la distribución a través del mercado, por principio, no es justa. Este enfoque considera entonces que la distribución y asignación deben mantenerse separadas, de tal forma que para que la primera sea justa es necesaria una política de transferencias (de propiedad, impuestos, recaudación para seguridad social, pago de servicios ambientales, etc.) que debería ser consensuada en función del beneficio social. Por otra parte, los precios determinados por la oferta y demanda serían el mecanismo base para llevar a cabo una asignación eficiente.

De esta manera, si la distribución se hace en función de valores monetarios se incurre en injusticia social al trasladar los costos de uso de recursos en los procesos productivos a la sociedad; sin embargo, si estos 
valores se utilizan para la asignación de recursos, los que pagarían estas externalidades serían los propietarios de los medios de producción.

Así, la distribución y la escala deberán estar respaldadas por el consenso social. En ello los elementos de decisión pueden ser diversos (indicadores biofísicos, sociales y económicos); entre éstos pueden considerarse ponderaciones de valores monetarios o numéricos para la toma de decisiones. Una vez resueltos estos dos problemas se podrán utilizar mecanismos de mercado para asignar recursos eficientemente. Estos tres elementos deberían ser tratados en forma interrelacionada ya que esto permitirá cumplir con los principios del desarrollo sustentable.

En resumen, para hacer operativo el enfoque de la economía ecológica y cumplir entonces con las metas del desarrollo sustentable, es indispensable tomar en cuenta tres factores: la escala sustentable, la distribución justa y la asignación eficiente de recursos; éstos tendrían que considerarse de manera integrada y en el orden siguiente: primero fijar los límites ecológicos de la escala sustentable, lo que pudiera lograrse a partir de la creación de políticas que aseguren que el crecimiento de la economía permanezca dentro de esos límites; segundo, establecer una distribución equitativa y justa a través de los instrumentos de política, que permita disminuir el grado de desigualdad; y tercero, utilizar los mecanismos del mercado para llevar a cabo la asignación (Costanza et al., 2014).

\subsection{Los valores de la sustentabilidad}

De acuerdo con lo sugerido por Costanza (2001) la valoración de los servicios de los ecosistemas basados en la eficiencia, justicia y sustentabilidad deberá estar fundamentada en tres tipos de valores:

1) El valor "E", basado en la eficiencia, se sustenta en las preferencias individuales que se suponen fijas. Se parte de que estas preferencias existen y su valor está dado por la disposición a pagar el bien o servicio dado; bajo esta consideración, la estimación más aproximada de lo que la gente quiere pagar sería lo que paga en el mercado real. En los casos donde no existe precio de mercado como en los servicios ambientales, éste puede ser estimado a partir de información arrojada con la aplicación de cuestionarios donde los individuos responden cuánto están dispuestos a pagar por el bien o servicio en cuestión.

2) El valor "F" (fair, o justo en español) está basado en la justicia, donde los sujetos expresan sus preferencias como miembros de la comunidad, no como individuos. Tendría que haber consenso sobre los valores que serían justos para todos los integrantes de la comunidad y para otras especies, tanto en el presente como en el futuro. En las discusiones para 
generar el consenso se incorpora información científica acerca de las consecuencias futuras de asignar tales valores.

3) El valor "S", basado en la sustentabilidad. Requeriría de una evaluación de la contribución a la sustentabilidad ecológica del recurso en cuestión; este valor está vinculado al rol físico, químico y biológico dentro del funcionamiento del sistema global, a largo plazo, por lo que es necesario contar con información científica acerca del funcionamiento del sistema global y construir un consenso al respecto. El individuo expresa sus preferencias como si fueran representativas del sistema entero (preferencias individuales, comunitarias y del resto de los seres), en vez de ser únicamente una expresión de las preferencias individuales actuales.

Con lo analizado hasta aquí se puede asumir que el valor de los servicios basado en la eficiencia económica implica menor dificultad para la toma de decisiones; sin embargo, en éste no necesariamente estarían reflejadas las preferencias de todos los miembros de la comunidad, por lo que sería más adecuado el valor basado en la justicia. Ahora bien, no obstante las bondades del valor F, se excluye el papel del sistema de soporte a la vida, es decir, el entorno donde tienen lugar las relaciones entre lo social y lo económico. El valor sustentable intenta interrelacionar en el largo plazo los aspectos físicos con los sociales y económicos a través del conocimiento, considerando las preferencias del sistema en su conjunto, lo que aseguraría la sustentabilidad en el largo plazo pues se cumpliría con las metas básicas referidas: escala sustentable, distribución justa y asignación eficiente.

\section{Conclusiones}

El desarrollo sustentable, más que una ciencia o técnica, es un principio ético-filosófico-normativo que pretende perpetuar la vida humana, de ahí que en sí mismo este paradigma no incorpore las condiciones para hacerse operativo por lo que requiere apoyarse en diversas disciplinas como la economía, de la que se desprenden básicamente dos enfoques: la economía ambiental y la economía ecológica.

La primera busca integrar los elementos del desarrollo sustentable a través del razonamiento tradicional de la economía neoclásica, mientras la segunda, aunque reconoce aportaciones de la anterior, la trasciende y propone una reestructuración conceptual. El paradigma de la economía ecológica basa sus propuestas en valores como la eficiencia, equidad, justicia y la capacidad de regeneración y asimilación del ecosistema global de forma sustentable.

La revisión teórica realizada permite sostener, con Jiménez-Herrero (2003), la existencia de una mayor compatibilidad de la economía eco- 
lógica con las metas del desarrollo sustentable; sin embargo, es evidente que dicho enfoque recurre también al uso de las herramientas de la economía ambiental como complemento de las propias. Así, la economía ecológica hace suya la propuesta de operativizar el desarrollo sustentable a través de la estimación del valor sustentable, cuya base son las preferencias del sistema en su conjunto (preferencias individuales, comunitarias y del resto de los seres). El uso del valor sustentable implicaría su integración al sistema de toma de decisiones y aseguraría la sustentabilidad a largo plazo.

El valor sustentable puede incluir los valores de eficiencia (E) y justicia (F), los cuales serían utilizados como instrumentos para fundamentar la decisión social (consenso), tanto en la distribución, entendida como el uso de instrumentos por parte del Estado para propiciar la equidad, como en la escala, entendida como la capacidad de carga, asimilación y recuperación del planeta. Por otra parte, la obtención del valor de la eficiencia en la asignación de recursos, se basaría en los mecanismos del mercado.

De esta forma, poner en práctica u operativizar el desarrollo sustentable implica considerar tres tipos de valores, uno desde las herramientas y principios de la economía ambiental (E) y dos desde la economía ecológica (F y $\mathrm{S}$ ); estos valores permitirían generar criterios que a su vez fundamentarían las decisiones para llegar a definir una escala sustentable, una distribución justa y una asignación eficiente de los recursos.

Así, es posible sostener que puede avanzarse hacia el desarrollo sustentable a través de la puesta en práctica de los instrumentos propuestos por ambas corrientes. Tanto los valores calculados en cifras monetarias como los arrojados por razonamientos lógicos, por la información científica y los derivados de consensos sociales, constituyen elementos indispensables y complementarios para una integral toma de decisiones.

En tal sentido, se propone trascender el falso dilema entre las posturas que descalifican las herramientas de uno u otro de ambos enfoques analizados. Es evidente que gran parte de los trabajos empíricos de valoración existentes se podrían enmarcar indistintamente en la economía ambiental o en la economía ecológica. De hecho, al revisarlos se advierte que la mayoría se queda en la generación del valor E, con lo cual se limita a una visión circunscrita a la perspectiva económica tradicional.

Esta limitante se puede resolver a través de la obtención de los valores S y F, mediante la construcción de modelos que los integren en las metas del desarrollo sustentable: escala sustentable, asignación eficiente y distribución justa. Esta integración posibilitará que el desarrollo de valores no se limite a la generación de un valor monetario o de ponderaciones por regiones, sino que se conformen modelos analíticos de soporte a la toma de decisiones, fundamentados en información científica y en el 
consenso social, de tal manera que sea posible contar con herramientas más adecuadas para avanzar en el camino del desarrollo sustentable.

\section{Bibliografía}

Aguilera-Klink, Federico y Vicent Alcántara (1994), De la economía ambiental a la economía ecológica, Icaria, Barcelona.

Azqueta, Diego (2002), Introducción a la economía ambiental, McGrawHill, Madrid.

Brundtland, Gro Harlem (1987), "Our common future: report of the 1987 World Commission on Environment and Development", United Nations, Oslo, pp. 1-59.

Carciofi, Ignacio y Diego Azqueta (2012), “Territorio, desarrollo tecnológico y gestión de recursos naturales renovables: el caso de la pesca”, Investigaciones Regionales, 23, Asociación Española de Ciencia Regional, Alcalá de Henares, pp. 145-170.

Castro e Silva, Manuela y Aurora Teixeira (2011), "A bibliometric account of the evolution of EE in the last two decades: is ecological economics (becoming) a post-normal science?", Ecological Economics, 70 (5), Elsevier, Cambridge, pp. 849-862.

Costanza, Robert (1991), Ecological economics: the science and management of sustainability, Columbia University Press, New York.

Costanza, Robert y Carl Folke (1997), "Valuing ecosystem services with efficiency fairness and sustainability as goals", en Gretchen C. Daily (ed.), Nature's services: societal dependence on natural ecosystems, Island Press, Washington, pp. 49-68.

Costanza, Robert (2001), "Visions, values, valuation, and the need for an ecological economics", BioScience, 51 (6), Elsevier, Cambridge, pp. 459-468.

Costanza, Robert, John Cumberland, Herman Daly, Robert Goodland y Richard Norgaard (2014), An introduction to ecological economics, St. Lucie Press, Boca Raton. 
Daly, Herman (1996), "Criterios operativos para el desarrollo sostenible", Eumed.net, <http://www.eumed.net/cursecon/textos/index. htm>, 12 de noviembre de 2013.

Derissen, Sandra y Uwe Latacz-Lohmann (2013), "What are Pes? A review of definitions and an extension”, Ecosystem Services, vol. 6, Elsevier, Cambridge, pp. 12-15.

Egoh, Benis, Belinda Reyers, Mathieu Rouget, Michael Bode y David Richardson (2009), "Spatial congruence between biodiversity and ecosystem services in South Africa", Biological Conservation, 142, Elsevier, Cambridge, pp. 553-562.

Ehrlich, Paul y Anne Ehrlich (1993), La explosión demográfica. El principal problema ecológico, Salvat, Barcelona.

Gasparatos, Alexandros y Anna Scolobig (2012), "Choosing the most appropriate sustainability assessment tool”, Ecological Economics, 80, Elsevier, Cambridge, pp. 1-7.

Georgescu-Roegen, Nicholas (1971), The entropy law and the economic process, Harvard University Press, Cambridge.

Gómez-Baggethun, Erick, Rudolf de Groot, Pedro Lomas, Carlos Montes (2010), "The history of ecosystem services in economic theory and practice: from early notions to markets and payment schemes", Ecological Economics, 69 (6), Elsevier, Cambridge, pp. 1209-1218.

Haro-Martínez, Alma y Cristina Taddei-Bringas (2010), "Valoración ambiental: aportaciones, alcances y limitaciones", Problemas del Desarrollo. Revista Latinoamericana de Economía, 41 (160), UnAM, México, pp. 209-221.

Herrmann-Pillath, Carsten (2011), "The evolutionary approach to entropy: reconciling Georgescu-Roegen's natural philosophy with the maximum entropy framework", Ecological Economics, 70 (4), Elsevier, Cambridge, pp. 606-616.

Illge, Lydia y Reimund Schwarze (2009), "A matter of opinion: how ecological and neoclassical environmental economists and think about sustainability and economics", Ecological Economics, 68 (3), Elsevier, Cambridge, pp. 594-604. 
Jiménez-Herrero, Luis (2003), "Cambio global, desarrollo sostenible y economía ecológica”, en Luis J. Jiménez-Herrero y Francisco J. Higón-Tamarit (eds.), Ecología y economía para un desarrollo sostenible, Universitat de Valencia, Valencia, pp. 13-62.

Kallis, Giorgos, Christian Kerschner y Joan Martínez-Alier (2012), "The economics of degrowth”, Ecological Economics, 84, Elsevier, Cambridge, pp. 172-180.

Kapp, William (1976), "El carácter de sistema abierto de la economía y sus implicaciones", en Federico Aguilera Klink y Vicent Alcántara (eds.), De la economía ambiental a la economía ecológica, Icaria, Barcelona, pp. 199-212.

Laurans, Yann y Laurent Mermet (2014), "Ecosystem services economic valuation, decision-support system or advocacy?", Ecosystem Services, 7, Elsevier, Cambridge, pp. 98-105.

Maynard, Simone, David James y Andrew Davidson (2014), "Determining the value of multiple ecosystem services in terms of community wellbeing: who should be the valuing agent?", Ecological Economics (s.p.i.).

Martínez-Alier, Joan (1996), "La economía ecológica como ecología humana”, ponencia presentada en el III Foro del Ajusco, 4-6 de septiembre, México.

Martínez-Alier, Joan, Jordi Roca-Jusmet y Jannette Sánchez (1998), Curso de economía ecológica, Programa de las Naciones Unidas para el Medio Ambiente-Oficina Regional para América Latina y el Caribe, México.

Martínez-Alier, Joan y Jordi Roca-Jusmet (2001), Economía ecológica y política ambiental, Fondo de Cultura Económica, México.

Munda, Giuseppe (1995), Multicriteria evaluation in a fuzzy environment: theroy and applications in ecological economics, Physica-Verlag, Heidelberg.

Naredo, José Manuel (1994), "Fundamentos de la economía ecológica”, en Federico Aguilera Klink y Vicent Alcántara (eds.), De la 
economía ambiental a la economía ecológica, Icaria, Barcelona, pp. 231-252.

Naredo, José Manuel (1996), Sistema de cuentas de los recursos naturales y el medio ambiente, ponencia presentada en III Congreso Nacional del Medio Ambiente, 25-29 de noviembre, Madrid.

Naredo, José Manuel (2001), "Economía y sostenibilidad: la economía ecológica en perspectiva”, Polis. Revista Académica Universidad Bolivariana, 1 (2), Universidad Bolivariana, Santiago de Chile, pp. 2-28.

Ness, Barry, Evelin Urbel-Piirsalu, Stefan Anderberg y Lennart Olsson (2007), "Categorising tools for sustainability assessment", Ecological Economics, 60, Elsevier, Cambridge, pp. 498-508.

Riechmann, Jorge (1995), "Desarrollo sostenible la lucha por la interpretación”, en José Manuel Naredo y Jorge Riechmann (eds.), De la economía a la ecología, Trotta, Madrid, pp. 11-35.

Schagner, Jan Philipp, Luke Brander, Joachim Maes y Volkmar Hartje (2013), "Mapping ecosystem services' values: current practice and future prospects", Ecosystem Services, vol. 4, Elsevier, Amsterdam, pp. 33-46.

Singh, Rajesh, H. R. Murty, S. K. Gupta y A. K. Dikshit (2009), "An overview of sustainability assessment methodologies", Ecological Indicators, 9 (2), Elsevier, Amsterdam, pp. 189-212.

Spash, Clive (2013), "Influencing the perception of what and who is important in ecological economics", Ecological Economics, vol. 89, Elsevier, Cambridge, pp. 204-209.

Tacconi, Luca (2012), "Redefining payments for environmental services", Ecological Economics, 73, Elsevier, Cambridge, pp. 29-36.

Tetreault, Darcy Víctor (2008), "Escuelas de pensamiento ecológico en las Ciencias Sociales", Estudios Sociales, 16 (32), Ciad, Hermosillo, pp. 228-263.

Van-Dyke, Fred (2008), Conservation biology. Foundations, concepts, applications, McGraw-Hill, New York. 
Venkatachalam, Lakshmanan (2007), "Environmental economics and ecological economics: Where they can converge?", Ecological Economics, 61 (2-3), Elsevier, Cambridge, pp. 550-558.

Willis, Kenneth, Kenneth Button y Peter Nijkamp (1999), Enviromental valuation, vol. I. Methods and anomalies, Edward Elgar Publishing, Cheltenham.

Recibido: 12 de septiembre de 2011. Reenviado: 14 de enero de 2013. Aceptado: 30 de septiembre de 2013.

Alma Angelina Haro-Martínez. Mexicana. Doctora en ciencias con especialidad en Desarrollo Regional por el Centro de Investigación en Alimentación y Desarrollo, A.c. Es profesora de la Universidad Estatal de Sonora y trabaja en diversos proyectos ambientales regionales. Entre sus publicaciones recientes destacan: "Valoración ambiental: aportaciones, alcances y limitaciones", Problemas del Desarrollo. Revista Latinoamericana de Economía, 41 (160), unam, México, pp. 209-221 (2010); "Responsabilidad social en el sistema vid de mesa de Sonora: de una responsabilidad inducida a una responsabilidad ética", ponencia presentada en el XVI International Symposium of Consorcio Internacional de Desarrollo Social, 27-31 de julio, Monterrey (2009).

Isabel Cristina Taddei-Bringas. Mexicana. Doctora en ciencias económicas por la Universidad Autónoma Metropolitana en México. Actualmente es investigadora titular del Departamento de Desarrollo Regional del Centro de Investigación en Alimentación y Desarrollo, A.c. Es miembro del Sistema Nacional de Investigadores. Su línea de investigación es organización industrial, mercados y cadenas productivas. Entre sus publicaciones recientes se encuentran "Patrones de consumo de carne en el noroeste de México", Revista Estudios Sociales, XX (2), CIAD, Hermosillo, pp. 73-96 (2012); "Análisis de competitividad de cuatro sistema-producto estatales de tilapia en México", Revista Estudios Sociales, 18 (35), CIAD, Hermosillo, pp. 165-207 (2010); "Valoración ambiental: aportaciones, alcances y limitaciones", Problemas del Desarrollo. Revista Latinoamericana de Economía, 41 (160), unam, México, pp. 209-221 (2010). 